\title{
The Effect of Kidney Biopsy on Glomerular Filtration Rate: A Frequent Patient Concern
}

\author{
Rakesh Dattani ${ }^{a} \quad$ Richard W. Corbett $^{\mathrm{a}} \quad$ Jack Galliford ${ }^{\mathrm{a}}$ Peter Hill ${ }^{\mathrm{a}}$ Tom Cairns ${ }^{\mathrm{a}}$ \\ H. Terence Cook ${ }^{b}$ Candice Roufosse ${ }^{b}$ Damien R. Ashby ${ }^{a}$ \\ aRenal and Transplant Centre, Hammersmith Hospital, Imperial College Healthcare NHS Trust, London, UK; \\ ${ }^{b}$ Department of Immunology and Inflammation, Centre for Inflammatory Diseases, Faculty of Medicine, Imperial \\ College, London, UK
}

\section{Keywords}

Renal biopsy · Glomerular filtration rate

\begin{abstract}
The effect of percutaneous kidney biopsy on glomerular filtration rate has never been identified, though it is frequently a concern raised by patients. Following a clinical interaction with an inquisitive patient undergoing her fifth biopsy, we attempted to estimate the effect using retrospective data. In a cohort of patients with stable kidney function undergoing transplant biopsy without clinical indication (as part of a surveillance programme) the effect of biopsy was observed as a step change in glomerular filtration rate. Reassuringly, the loss of glomerular filtration rate resulting from a biopsy, has a 1-sided $95 \%$ confidence interval of $<1.4 \mathrm{~mL} /$ $\min$.

(C) 2020 S. Karger AG, Basel
\end{abstract}

\section{Introduction}

Since their first description in the 1950s [1], percutaneous renal biopsies have become central to the management of patients with renal dysfunction [2], remaining essential for the histopathological correlation of disease in both native and transplanted kidneys [3, 4]. Progressive refinements in technique have led to an improved safety profile and as a result a lower threshold for biopsy; however, as with any procedure, benefits must be weighed against risks. While the incidence of minor complications is dependent upon the methodology used for detection [2], major complications, such as those where bleeding requires intervention, are rare [5].

Unknown, however, is the effect of biopsy on glomerular filtration rate (GFR), though this is often a patient concern. Given the small size of a biopsy sample, and in particular the number of glomeruli usually observed, one imagines the effect to be negligible; however, it seems selfevident that the effect cannot be zero, and no work to date has attempted to quantify this. The effect of biopsy on GFR is usually confounded by the indication for biopsy, so that the effects of disease and procedure cannot be distinguished. In contrast, surveillance ("protocol") transplant renal biopsies are performed in the absence of clinical indication [6] and therefore provide a unique opportunity to examine the effect of biopsy alone. In a single-centre retrospective study, we aimed to examine GFR changes in a cohort of patients with stable transplant function, from 6 months before until 12 months after surveillance biopsy, with the aim of determining the effect of kidney biopsy on GFR. karger@karger.com www.karger.com/ajn

(C) 2020 S. Karger AG, Base

Karger"
Rakesh Dattani

Imperial College Renal and Transplant Centre

Hammersmith Hospital

Du Cane Road, London (UK)

rakesh.dattani@nhs.net 


\section{Case Report}

A 33-year-old Caucasian female requested an early clinic review, complaining of ankle swelling. She had first presented aged 19 , with polyarthralgia and heavy proteinuria, with low C4 alongside raised double-stranded DNA. Her kidney biopsy demonstrated class IV lupus nephritis, and she was treated with cyclophosphamide and rituximab. Over the next 6 years there were several nephrotic relapses, treated with cyclophosphamide, rituximab, or eculizumab, often informed by further biopsy tests, and she received maintenance treatments including tacrolimus and mycophenolate mofetil. She then entered a prolonged remission, on low dose mycophenolate mofetil, until this request for clinic review.

She appeared to have relapsed again, with heavy proteinuria, but was disappointed at the suggestion of another biopsy. This would be her fifth, and rather than pain or inconvenience, she was mostly concerned about the cumulative effect of repeated biopsy ("surely it damages my kidneys every time we remove a bit"). She was unsatisfied by the explanation that any such effect must be very small ("but it cannot be zero") and unimpressed by the lack of actual data in these explanations ("has not anyone ever looked?"). She went ahead with the biopsy, and we agreed to take her concern seriously, and at least attempt to provide a data-derived answer.

\section{Methods}

In a mixed ethnicity renal centre in London, $\mathrm{UK}$, all patients undergoing kidney biopsy over a 5 year period between 2008 and 2012 were retrospectively identified. To avoid confounding the effect on GFR by underlying kidney disease, only transplant surveillance biopsies, over 7 months post-transplantation, were included. To ensure the absence of indication, patients with GFR changing by $>10 \mathrm{~mL} / \mathrm{min}$ in the interval prior to biopsy and those with intercurrent illness (indicated by $>24$ blood samples in any 3 -month period) were excluded. All Cr data from 6 months prior to 12 months post-biopsy were collected. For each patient the median Cr over each quarter (3-month period) was used to calculate GFR using the 4-variable MDRD equation. GFR differences between intervals were compared using the $t$ test, and 1-sided confidence intervals were calculated, giving an upper limit to the effect of biopsy on GFR. The research board determined that formal ethical review was unnecessary.

\section{Results}

From 357 surveillance biopsies $>7$ months after transplantation, complete data sets without intercurrent illness were available for 272 procedures, performed in 258 patients (aged 20-75, 58.0\% male) which were included in the analysis. There were no serious biopsy complications, but 3 patients with visible haematuria following biopsy were excluded. Surveillance biopsies were performed at median (IQR) 16 (11-46) months post-transplantation, at a time when mean $( \pm$ SE) GFR was $59.4( \pm 1.1) \mathrm{mL} /$ $\min$.
All samples were taken with $18 \mathrm{G}$ needles, and the mean $( \pm \mathrm{SD})$ sample size was $1.6( \pm 0.7)$ biopsy cores and $14.0( \pm 6.4)$ glomeruli. Average tubular atrophy was $16 \%$, and there was no other finding in 240 samples (88.2\%), the others showing minor histological changes including subthreshold features of cellular rejection. Cases were not excluded from analysis on the basis of histopathological findings, as positive findings would be expected to be evenly distributed throughout all time periods (whether observed by biopsy or not), and any effect on GFR would therefore be consistent throughout the analysis period.

Figure 1 shows mean GFR from 6 months before to 12 months after the biopsy, split into quarterly intervals (Q1-Q6) with the biopsy taking place between Q2 and Q3. Mean GFR in Q3 was lower than Q2 $(58.1 \pm 1.1$ vs. $59.4 \pm 1.1 \mathrm{~mL} / \mathrm{min}, p=0.001)$. There was also a pattern of gradual GFR decline across the whole period of observation, but the GFR change at the biopsy interval (Q2-3) was also greater than that at other intervals $(-1.27 \pm 0.38$ vs. $-0.34 \pm 0.17 \mathrm{~mL} / \mathrm{min}, p=0.039)$, suggesting a step change at this time point. These changes were mirrored by parallel changes in $\mathrm{Cr}$, rising from 119.7 to 127.8 $\mu \mathrm{mol} / \mathrm{L}$ across the observation period.

To estimate the size of the biopsy effect, GFR trajectory post-biopsy was calculated from intervals Q3 to Q6, and a trajectory with the same gradient $(-0.37$ per quarter) passing through GFR at Q1, used to estimate the prebiopsy trajectory (Fig. 1). Q1 was used rather than Q2 to avoid bias due to regression to the mean, since "surveillance" selects patients for Q1-2 stability. The gradient was sense-checked against a separate control cohort (in 1,395 transplant patients [mean age $55.3 \pm 12.6,60.4 \%$ male] not experiencing transplant failure with $\mathrm{Cr}$ readings 1 year apart, GFR fell from $51.7 \pm 0.5$ to $50.0 \pm 0.5$ $\mathrm{mL} / \mathrm{min}$ over 12 months, giving a mean $[ \pm \mathrm{SE}]$ GFR change per quarter of $-0.42 \pm 0.08 \mathrm{~mL} / \mathrm{min}$, Figure 1 inset figure). Thus GFR loss due to the biopsy, defined by the vertical distance between these 2 lines, was $0.77 \mathrm{~mL} / \mathrm{min}$, with a 1-sided $95 \%$ confidence interval of $<1.41 \mathrm{~mL} / \mathrm{min}$.

Compared to the rest of the group, those losing the most function after biopsy (in the highest tertile of GFR loss) had their biopsy performed earlier post-transplantation (24.8 vs. 33.3 months, $p=0.027)$ and had higher initial GFR (64.2 vs. $57.1 \mathrm{~mL} / \mathrm{min}$ during Q1, $p=0.004$ ) with tendencies towards less chronic change, including tubular atrophy ( 13.7 vs. $17.9 \%, p=0.066)$ and glomerular obsolescence (13.2 vs. $16.7 \%$ of glomeruli, $p=0.160$ ). No differential effect on GFR loss was observed related to biopsy size (number of cores or number of glomeruli sampled), age, gender, or ethnicity. 


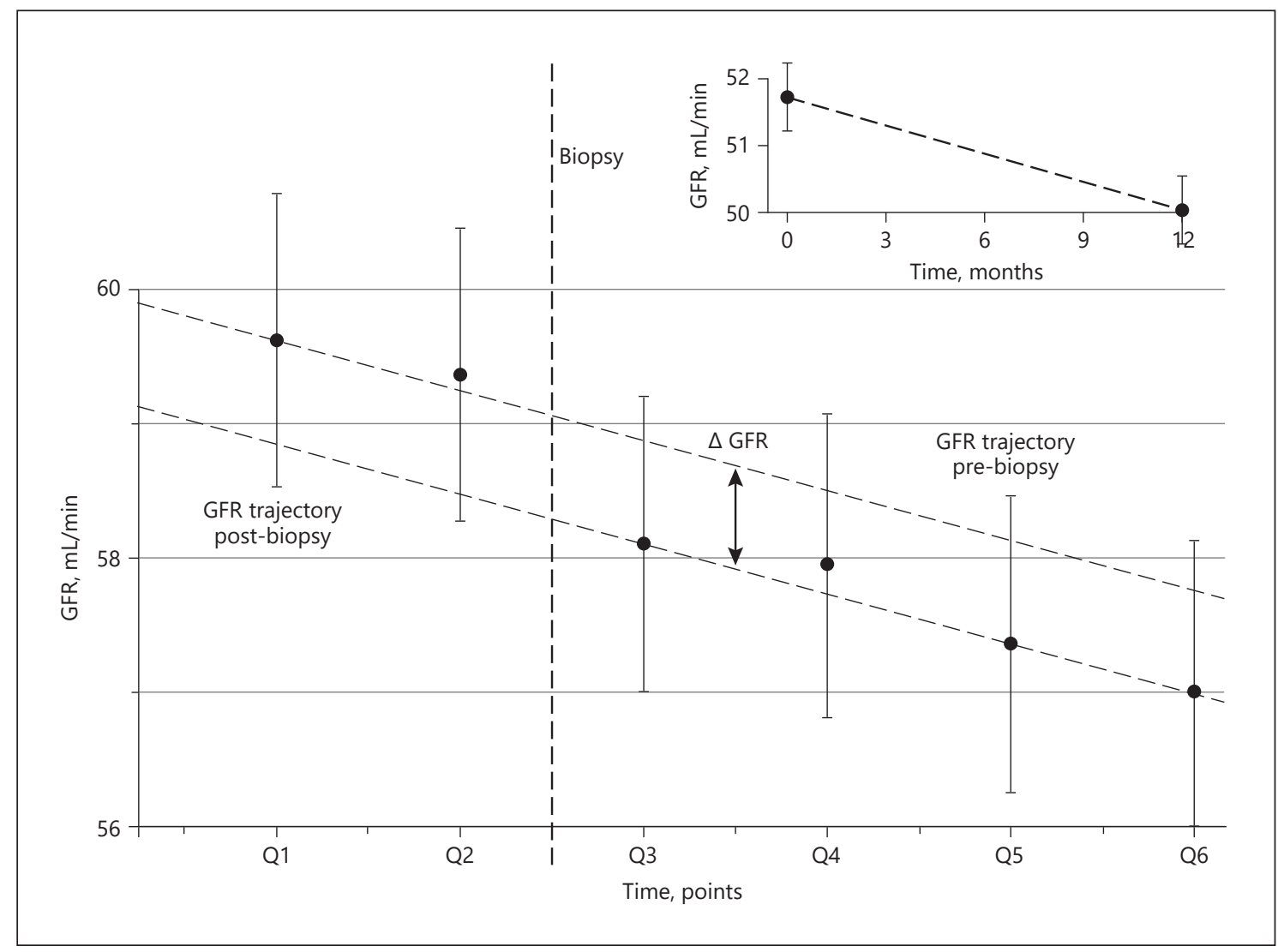

Fig. 1. Transplant function before and after biopsy. Mean ( \pm SE) GFR by quarter (3-month period), with biopsy between Q2 and Q3. Trajectory of GFR decline is calculated from post-biopsy quarters Q3 to Q6. The same gradient is used for the trajectory pre-biopsy passing through the GFR in Q1. GFR change due to the biopsy is calculated as the vertical difference between these lines. Inset Mean $( \pm$ SE) GFR in 1,395 transplant patients not experiencing transplant failure at time 0 and 12 months. GFR, glomerular filtration rate.

\section{Case Conclusion}

The patient's fifth biopsy showed podocytopathy with minor glomerular and tubulointerstitial scarring, and scant evidence of immune-complex pathology. She was treated with rituximab, and once again achieved remission over a few weeks. She was reassured to learn that the loss of GFR with each biopsy has an upper limit of $1.4 \mathrm{~mL} /$ $\min$.

\section{Discussion}

When consent is sought for percutaneous kidney biopsy, patients often inquire about the effect of the biopsy on kidney function. This study demonstrates a detectable change in GFR at the time of kidney biopsy and to our knowledge is the first study to quantify this effect. Along with the risk of a bleeding complication, this effect needs to be weighed against the possible improvement in prognosis as a result of a directed therapy, but it appears to be small. Although the GFR loss is estimated with low accuracy, the 1 -sided $95 \%$ confidence interval of $<1.4 \mathrm{~mL} / \mathrm{min}$ should be reassuring to most patients.

Plausibility of the estimate of GFR may be considered by comparison with single-nephron GFR, estimated to be $80( \pm 40) \mathrm{nL} / \mathrm{min}$ in live donors [7], which suggests that a reduction of $0.7 \mathrm{~mL} / \mathrm{min}$ would arise from the disruption of 8,000 nephrons. This is far greater than the 14 glomeruli seen in the average biopsy sample. However, 2 biopsy cores each causing fibrosis up to $4 \mathrm{~mm}$ in diameter, would disrupt a total cortical volume of $0.5 \mathrm{~cm}^{3}$, encompassing approximately 2,500 nephrons [8]. This is still $<8,000$, but since nephrons distant from the biopsy may also be damaged, for example by collecting tubule fibrosis 
or local haemodynamic effects, this estimate seems plausible. Equally, given that a haematoma can be detected on imaging in over $80 \%$ of patients undergoing a percutaneous kidney biopsy $[9,10]$, local compression may also play a role. Effects beyond the biopsy core itself may explain the finding that glomerular number and core size were not associated with the magnitude of GFR loss.

This study has a number of weaknesses which may limit the conclusions drawn. In particular, the sample size is small when one considers the unfavourable signal-tonoise ratio inherent in measuring small changes in GFR. For this reason, larger patient numbers are preferable to an approach using more accurate GFR measurement, such as iothalamate clearance, in a smaller number of patients. To reduce noise, it is tempting to exclude patients on the basis of histological findings; however, since the biopsies were all performed without clinical indication, the same histological findings would be present but unobserved during other time intervals and in control patients, so their exclusion would introduce bias. All histological findings were therefore included, though efforts were made to ensure that biopsies were performed genuinely for surveillance, without clinical indication. Therefore, although the sample size gives rise to a wide confidence interval, the conclusion that a small but detectable loss of GFR occurs seems justified.

Surveillance transplant biopsies offer a unique opportunity to examine the effect of biopsy in patients with stable kidney function, but the results are also of relevance to the biopsy of native kidneys. The small reduction in
GFR, with a 1-sided $95 \%$ confidence interval of $<1.4 \mathrm{~mL} /$ min, should be helpful in informing both patients and clinicians when considering kidney biopsy.

\section{Statement of Ethics}

The research board determined that formal ethical review was unnecessary. The patient discussed in the care report has given her written informed consent.

\section{Conflict of Interest Statement}

This study was supported by an NIHR Academic Clinical Fellowship. D.A. has received a speaker honorarium from Fibrogen. P.H. has received a speaker honorarium from Sanofi. The work was supported by the NIHR Imperial Biomedical Research Centre. The results presented in this paper have not been published previously in whole or part, except in abstract format.

\section{Funding Sources}

The authors did not receive any funding.

\section{Author Contributions}

The study was conceived by D.A. and conducted by R.D. Data were analysed by D.A. and R.C. with suggestions from other authors. The manuscript was written by R.D., edited by R.C. and D.A., and approved by all authors.

\section{References}

1 Iversen P, Brun C. Aspiration biopsy of the kidney. Am J Med. 1951 Sep;11(3):324-30.

2 Hogan JJ, Mocanu M, Berns JS. The native kidney biopsy: update and evidence for best practice. Clin J Am Soc Nephrol. 2016;11(2): 354-62.

3 Dhaun N, Bellamy CO, Cattran DC, Kluth DC. Utility of renal biopsy in the clinical management of renal disease. Kidney Int. 2014; 85(5):1039-48.

4 Williams WW, Taheri D, Tolkoff-Rubin N, Colvin RB. Clinical role of the renal transplant biopsy. Nat Rev Nephrol. 2012;8(2): $110-21$.
5 Lubas A, Wojtecka A, Smoszna J, Koziński P, Frankowska E, Niemczyk S. Hemodynamic characteristics and the occurrence of renal biopsy-related arteriovenous fistulas in native kidneys. Int Urol Nephrol. 2016;48(10):166773.

6 Henderson LK, Nankivell BJ, Chapman JR. Surveillance protocol kidney transplant biopsies: their evolving role in clinical practice. Am J Transplant. 2011;11(8):1570-5.

7 Denic A, Mathew J, Lerman LO, Lieske JC, Larson JJ, Alexander MP, et al. Single-nephron glomerular filtration rate in healthy adults. N Engl J Med. 2017;376(24):2349-57.
8 Charlton JR, Abitbol CL. Can renal biopsy be used to estimate total nephron number? Clin J Am Soc Nephrol. 2017;12(4):553-5.

9 Ralls PW, Barakos JA, Kaptein EM, Friedman PE, Fouladian G, Boswell WD, et al. Renal biopsy-related hemorrhage: frequency and comparison of CT and sonography. J Comput Assist Tomogr. 1987;11(6):1031-4.

10 Rosenbaum R, Hoffsten PE, Stanley RJ, Klahr S. Use of computerized tomography to diagnose complications of percutaneous renal biopsy. Kidney Int. 1978;14(1):87-92. 